\title{
Développer localement une pratique collaborative centrée sur les enseignements SHS pour des étudiants en sciences et techniques
}

\author{
Alain Bernard ${ }^{1}$, Sandrine de Montgolfier ${ }^{2}$ et Camille Roux-Goupille ${ }^{3}$ \\ ${ }^{1}$ Université Paris-Est Créteil et ESPE de l'académie de Créteil, Centre A. Koyré, France \\ ${ }^{2}$ Université Paris-Est Créteil et ESPE de l'académie de Créteil, IRIS, France \\ ${ }^{3}$ Université Paris-Est Créteil, UFR de sciences et techniques, STEF, France
}

\begin{abstract}
Résumé. Le premier objet de cet article est de présenter et décrire l'expérience locale d'une communauté ouverte d'enseignants partageant une problématique commune: la mise en place et le développement d'enseignements de sciences humaines et sociales pour des étudiants de sciences et techniques. Cette description attachée au fonctionnement concret de telles « micro » communautés constitue, en soi, une originalité de notre approche. En effet, alors qu'elles sont très probablement nombreuses dans les universités françaises, elles sont finalement très peu présentées et analysées dans leur dynamique collégiale et évolutive. D'autre part il s'agit de montrer que la théorie des communautés de pratique constitue un cadre très pertinent pour analyser a posteriori cette expérience locale et mieux en comprendre les enjeux et conditions. Ce cadre théorique nous permet finalement de tirer des conclusions sur l'avenir possible de cette initiative locale par une analyse des conditions qui lui permettent encore d'évoluer dont l'une d'elle est d'adosser la collaboration en vu de construire de nouveaux enseignements en SHS à des projets de recherche.
\end{abstract}

\section{Introduction}

Déterminer les caractéristiques communes des pratiques d'enseignement des sciences humaines et sociales dans des contextes de formation en sciences et techniques et en mesurer la généralité mériteraient une enquête fine et généralisée qui n'a, à notre connaissance, été réalisée que, de manière limitée en France, dans des secteurs très particuliers. C'est ainsi le cas du domaine de la formation des futurs médecins (Bonah 2006) ou celui de la formation des enseignants et seulement pour ce qui concerne l'épistémologie et l'histoire des sciences (Bernard and Laubé 2006). Concernant les enseignements de sciences humaines en médecine et santé, (Bonah 2006) montre que des communautés

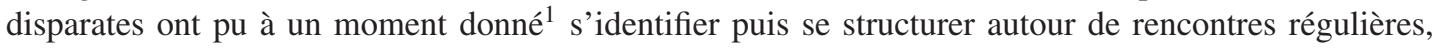
comme celles qu'organise dorénavant le collège des enseignants SHS en médecine et santé. Ces communautés se sont donné des objets qui ont pour eux une valeur forte d'identification collective et de réflexion continue, à l'exemple de la rédaction du manuel de SHS pour médecine et santé (Collège des enseignants de SHS en médecine et santé 2011). Pour ce qui concerne spécifiquement

\footnotetext{
1 En 1992/3.
}

This is an Open Access article distributed under the terms of the Creative Commons Attribution License 4.0, which permits unrestricted use, distribution, and reproduction in any medium, provided the original work is properly cited. 
la place de l'épistémologie et de l'histoire des sciences et des techniques dans la formation des enseignants, les années 2005-9 ont vu se structurer, puis disparaître, une communauté informelle d'enseignants chercheurs impliqués dans la formation ${ }^{2}$ : Là encore, cette structuration éphémère sans institutionnalisation forte, a néanmoins permis des rencontres et des publications qui ont fait vivre et progresser un certain nombre de problématiques ou permis des innovations pédagogiques, comme en témoigne plusieurs articles de ce volume (Husson et alii, Laubé et alii, Guedj).

A côté de ces exemples de coopération qui ont acquis une visibilité suffisante pour avoir laissé quelques productions, car elles avaient un caractère national, il en existe beaucoup d'autres qui se développent à une échelle locale, de manière plus ou moins formalisée. Ce caractère localisé rend peu lisibles ces expériences dans la mesure où elles ne sont que très rarement présentées et discutées, si ce n'est de manière trop confidentielle, ou alors sans que la dynamique du groupe concerné ne soit décrite en détail. Le présent article propose ainsi de s'attacher à cette échelle très locale, en traitant d'une coopération concernant un nombre restreint d'enseignants chercheurs et localisée sur le périmètre d'une université (l'UPEC). Cette communauté, dont les auteurs font partie, s'est développée en étant grosso modo centrée, sur l'instauration et le développement collégial d'enseignements en SHS s'adressant à des étudiants en sciences et techniques de tous niveaux. Le présent article a ainsi été élaboré à la lumière des remarques et réflexions des enseignants engagés dans ces pratiques mutualisées ${ }^{3}$.

Notre première partie expose un exemple centré autour d'une mutualisation d'expériences d'enseignement d'histoire des sciences et des techniques dans des formations de niveau master pour de futurs enseignants ${ }^{4}$. Cet exposé liminaire peut être vu comme un témoignage informé et autoréflexif.

Dans la partie suivante, nous proposons de montrer que la théorie des communautés de pratique est un cadre d'analyse pertinent pour caractériser cette expérience de mutualisation et en comprendre rétrospectivement les conditions d'émergence.

Enfin, dans la troisième et dernière partie nous mettons à profit ce même cadre théorique pour son intérêt prospectif, afin d'étudier d'une part les possibilités d'évolution de ce travail compte tenu de la situation actuelle, qui voit monter en force la demande pour ce type de cours; et d'autre part pour mieux cerner une des conditions primordiales de ce travail, qui était son association à un projet de recherche.

\section{Notre cas d'étude : fédérer la réflexion sur des cours d'histoire et d'épistémologie des sciences au niveau master pour de futurs enseignants}

\subsection{Un contexte favorable au développement d'une communauté évolutive}

La coopération que nous évoquerons ici concerne trois formations différentes en histoire et épistémologie des sciences pour des étudiants de master se destinant à l'enseignement, ces formations ayant donné lieu à trois séances différentes du séminaire « sciences et techniques en interférence ${ }^{5}$, accompagnée pour l'une d'entre elles d'un travail d'écriture important sur le carnet de recherche associé. Ces rencontres permettant de faire un bilan critique de la mise en place de ces formations, et de la manière de les faire évoluer, ont donc été rendues possible par l'existence même de ce séminaire. Cette existence doit elle-même être rapportée à la longue gestation qui a conduit à la construction de l'actuelle

\footnotetext{
${ }^{2}$ Le groupe a existé sous l'acronyme ReForEHST (Recherche et Formation sur l'Epistémologie et l'Histoire des Sciences et des Techniques). Voir par exemple (Guedj 2006).

3 Voir le programme de la séance de février 2014 du séminaire « sciences et techniques en interférences 》: http://interferences.hypotheses.org/625.

${ }^{4}$ Cette partie correspond à la présentation qui a été faite par l'un d'entre nous lors du colloque de février 2013. Voir le résumé : http://shst2013-upec.sciencesconf.org/9684.

5 http://interferences.hypotheses.org
} 
ÉSPÉ de l'académie de Créteil. Avant même en effet que la constitution des ÉSPÉ 6 ne devienne un enjeu national fin 2012, l'équipe présidentielle de l'UPEC d'alors avait proposé, dans le contexte de crise qui accompagnait la première réforme dite de la «mastérisation » des formations d'enseignants (2008-9), une réflexion de fond pour restructurer l'institut universitaire de formation des maîtres (IUFM) vers une nouvelle composante. Cette nouvelle composante devait avoir, entre autres vocations, la formation à l'enseignement supérieur ainsi qu'une politique de recherche mieux identifiée. Dans ce contexte, il était demandé aux enseignants chercheurs de l'institut de faire un effort particulier pour réfléchir à une structuration possible du programme de recherche de cette nouvelle école. De là est né en 2011 un des axes de ce programme de recherche, animé par une équipe d'enseignants chercheurs de l'ex-IUFM, dont deux d'entre nous faisions partie, et une première problématique évolutive qui a fait ensuite l'objet de plusieurs révisions ${ }^{7}$. A partir de juillet 2012, ce groupe s'est doté d'un carnet de recherche qui servira ici d'archive de la réflexion menée au sein du groupe ${ }^{8}$.

Puisque l'idée du programme initial était de se tourner entre autres thématiques vers des questions de réflexion sur l'enseignement supérieur ${ }^{9}$, il comporte un sous-axe de travail sur les enseignements de sciences humaines. Il implique aussi que le groupe ne soit pas considéré comme limité aux seuls membres fondateurs, mais ouvert à d'autres composantes et d'autres collègues intéressés par cette problématique de recherche. Le colloque de février 2013 faisait précisément partie du programme visant à étendre et développer cette coopération et à l'inscrire dans une perspective comparatiste entre différents domaines de formation professionnelle. Plus généralement, l'idée de bâtir un séminaire régulier faisait partie du programme général rendant possible le développement de ces travaux.

L'autre partie de ce programme de travail initial visait à développer un mode de mutualisation et de développement des enseignements de sciences humaines conduits et développés par les membres du groupe ${ }^{10}$. Même si ce projet n'a pas pour l'instant abouti au but défini initialement, à savoir la construction d'un outil collaboratif d'inventaire de formations, le point important est, ici, qu'il a donné lieu à plusieurs rencontres ayant eu ultérieurement un effet sur le développement des formations en question, sur l'élargissement du cercle des participants à cette réflexion, enfin à des idées nouvelles qui n'étaient pas contenues dans le projet initial. Il doit donc être examiné pour les effets de bifurcations et de développement qu'il a produit plus que sur son résultat escompté.

Si l'on se restreint pour l'instant à la seule question du développement du groupe des collègues impliqués dans cette réflexion, on peut noter que la première séance du séminaire 2012-13 en septembre $2012^{11}$ a permis d'associer une des cosignataires de cet article, qui est devenue ensuite membre du groupe. La seconde séance, en novembre 2012, a permis d'associer plusieurs enseignants intervenant dans le master dont faisait partie la formation discutée ${ }^{12}$ : si cette discussion n'a pas débouché concrètement sur une coopération plus approfondie, l'idée en a été lancée en constatant que les thèmes traités dans le cours examiné méritaient d'être repris dans d'autres parties de la formation. Enfin la

\footnotetext{
6 Écoles Supérieures du Professorat et de l'Éducation.

${ }^{7}$ Pour l'état primitif de cette problématique, voir le billet suivant, daté de juillet 2012 mais renvoyant à une réflexion courant sur fin 2011 et début $2012:$ http://interferences.hypotheses.org/42

${ }^{8}$ Dans la suite, ce carnet est simplement appelé « carnet STI » et on ne donnera que le numéro des billets cités. Le billet $n$ est donc obtenu à partir de l'URL http: //interferences . hypotheses.org/n

${ }^{9}$ Voir l'axe 2 de travail du programme énoncé en juillet 2012 (note précédente), qui portait sur « la conception d'enseignements ou de ressources pour l'enseignement au niveau universitaires portant sur les sciences et techniques sous une perspective de sciences humaines (philosophie et épistémologie, éthique et histoire, didactique, sociologie ... ) : enjeux, modalités, possibilité d'une approche comparatiste, place des environnements numériques ».

${ }^{10}$ Le détail de ce projet initial est énoncé dans le billet 37 du carnet STI.

${ }^{11}$ Elle a permis la discussion autour d'un enseignement d'épistémologie et d'histoire des techniques inscrit dans le master de formations des enseignants de lycées technologiques et professionnels (MFTIGS) porté par l'IUFM de Créteil. Voir l'annonce dans le billet 125 et le bilan dans les billets 236 et 294 du carnet STI.

12 Il s'agissait d'un enseignement d'histoire des sciences de la vie et de la terre, inscrit dans le master préparant les futurs enseignants de SVT, commun à l'UPEC et à l'université Paris 7. Carnet STI, billet 273.
} 
troisième séance ${ }^{13}$ a permis d'associer un collègue qui était intervenu dans un master pour remplacer dans le cadre d'une vacation l'un d'entre nous pendant l'année 2011-12 .

On voit donc à ces trois exemples comment ce type de réunion, par leur existence même et à condition d'être suffisamment publicisé d'une part, et d'avoir un objectif défini d'autre part, peut permettre l'établissement d'une communauté regroupant des enseignants intéressés, d'associer de nouveaux membres et de diversifier les modes d'interaction et de coopération et d'engagement dans des projets d'enseignements.

Dans les deux parties suivantes, nous examinons plus précisément les effets de cette collaboration notamment sur le développement de nouveaux outils de travail.

\subsection{Le développement puis l'usage pédagogique d'un outil de visualisation des cours concernés}

Un des premiers résultats des travaux de la première séance évoquée ci-dessus, a été de développer la réflexion sur le moyen concret de visualiser de manière synthétique le déroulement du module qui était examiné. Il s'agissait de pouvoir décrire l'enchaînement des séances et, surtout, celui des travaux demandés aux étudiants dans ce cadre (Annexe $1[\underline{x}])^{15}$. Par exemple, dans le cas évoqué, le schéma permet de constater que la succession des travaux d'étudiants impliquait deux ensembles parallèles unissant les cours d'un côté et une activité liée à une sortie au CNAM, et de l'autre les travaux dirigés. Les travaux dirigés avaient pour fonction de suivre sur l'ensemble du module la construction d'un rapport sur un sujet d'épistémologie et d'histoire des sciences choisi par les étudiants. Ce schéma créé a posteriori permet de bien visualiser les interactions entre les différentes parties du module et leur complémentarité.

Même si ce module n'a pas été pérenne étant donné l'évolution drastique du cadre de formation des enseignants depuis 2012, on peut suggérer ce qu'aurait pu être son usage auprès des étudiants et des collaborateurs du master. Aux premiers, il aurait permis de mieux faire comprendre les objectifs du module, les différentes étapes, les travaux à rendre, les interactions entre les activités. Pour les seconds, cet outil nous semblerait précieux pour communiquer entre les différents collaborateurs du module afin de pouvoir travailler en parallèle dans les TD avec un même objectif et de pouvoir faire des points d'étapes. Enfin, et peut-être le plus important, cet outil, présenté aux enseignants du master, pourrait nous aider à mieux communiquer sur les interactions possibles avec les autres modules du semestre et de l'année de ce master afin de créer des ponts. Autrement dit il pourrait s'ajouter aux outils à utiliser pour mieux discuter des évolutions du master dans sa globalité dans le cadre de la création du master MEEF et pourrait être généralisé au niveau local pour communiquer entre enseignants.

Simultanément, nous avons théorisé cette façon de constituer un « critère heuristique » ${ }^{16}$ en parlant, au sujet de ce type de représentation, «d'organisation synoptique ». L'élaboration de cette notion a eu ensuite un impact intéressant sur la troisième séance qui était consacrée à un autre cours d'histoire des sciences analysé dans son évolution sur deux années différentes: il a donc paru intéressant de restituer le déroulement d'une manière semblable et pour les deux années consécutives. Les figures obtenues

\footnotetext{
13 Elle était consacrée à un enseignement d'histoire des mathématiques, inscrit dans une spécialité mathématiques-enseignement commune à trois masters de mathématiques sur les universités UPEC, UPEM et Paris 13. Voir carnet STI, billet 290.

14 On verra plus loin que ce même collègue a ensuite participé à une formation pour doctorants où une partie du matériel élaboré pendant cette séance a été exploitée.

15 Les travaux en question étaient la préparation de compte rendu écrits, de présentations orales, de dossiers. L'ensemble des tableaux en annexes est présenté dans le billet 670 du carnet STI.

${ }^{16}$ La notion de « critère heuristique » a été élaborée en général, pour désigner toute manière synthétique de communiquer un aspect des formations discutées, qui puisse faire l'objet d'une transposition à d'autres formations, et notamment à des formations nouvelles, d'où le terme « heuristique ». Pour des explications plus détaillées, voir la page « critère » du carnet STI : http://interferences .hypotheses .org/comp
} 
(annexes $2[\underline{x}]$ et $3[\underline{x}]$ ) illustrent clairement une structure en deux parties, la première constituée d'un enchaînement relativement classique de séances associant une partie de cours et une autre de travaux dirigés, même si la présentation diffère et est plus détaillée pour l'année 2011-12. Là encore, ce qu'on a fait figurer est non seulement l'enchaînement des contenus travaillés sur chaque séance (ou le principe de travail pour les dernières séances, qui se déroulaient en partie à la BnF), mais également l'enchaînement des tâches demandées aux étudiants.

Sans entrer ici dans le détail d'un commentaire détaillé de ces deux organisations synoptiques, on peut remarquer que leur comparaison révèle une parenté de conception frappante ; parenté qui serait encore mieux soulignée si on avait documenté de la même manière la première édition du cours en 2010-11, qui suivait un principe très différent du schéma adopté en 2011-12 ainsi que globalement l'esprit d'une pédagogie par projets. Mais les deux schémas (annexes $2[\underline{\mathrm{x}}]$ et $3[\underline{\mathrm{x}}]$ ) renseignent non seulement sur une temporalité relative de chaque partie un peu différente, mais surtout sur un degré de maturité différent. En particulier, le schéma « novice» de 2012-13 ${ }^{17}$ est moins renseigné que le schéma de l'année précédente notamment en ce qui concerne les consignes précises données d'un cours à l'autre. Cette différence renseigne indirectement sur la portée explicative de telles schématisations: la comparaison a permis d'affiner la compréhension rétrospective de ce qui était en jeu dans ce type de cours et ainsi de passer d'une imitation encore novice vers une appropriation plus complète du travail attendu des étudiants.

Cette dynamique positive n'a pu être poursuivie car le cours a été purement et simplement supprimé après 2012, en raison d'une nouvelle réforme globale de la formation des enseignants qui ne laissait plus de place à ce type d'enseignement en première année de master ${ }^{18}$. Il n'est donc pas possible d'examiner, là encore, comment les leçons de la comparaison auraient pu être tirées pour une nouvelle édition du cours $^{19}$. Par contre, il faut prendre garde à la date d'élaboration du premier schéma, en fait postérieure au second: elle a été occasionnée par l'intervention de l'enseignant dans une formation à l'enseignement supérieur pour doctorants ${ }^{20}$, pour laquelle il est apparu intéressant de faire réfléchir les doctorants sur les compétences travaillées à travers ce cours. Le schéma primitif qu'on trouve en annexe 2 [x] a en fait été élaboré pour ce cours et il a donné lieu à un travail de réflexion proposé aux étudiants, conclu par un schéma mettant en évidence les compétences travaillés à partir de ce type de tâches et d'organisation: ce schéma « augmenté» se trouve en annexe 4 [ $\underline{x}]$.

Au total, on est ainsi passé d'un schéma à valeur explicative, conçu pour un travail de réflexion entre pairs, à un schéma à valeur initiatrice et pédagogique utilisable dans une formation pour jeunes enseignants à l'université. Mais il n'y a pas de solution de continuité de l'un à l'autre: du point de vue de la pratique partagée, il signifie simplement que le travail mené en commun lors des séances du séminaire a débouché sur des documents utilisables à l'extérieur du premier groupe d'enseignants qui y a travaillé, et que les étudiants ont été placés en position d'observateurs périphériques mais réflexifs par rapport à ce travail. La plupart de ces étudiants contractuels ne sont pas encore en position, sauf exception, de concevoir un cours dans son entier: l'auraient ils été, il aurait été possible de leur suggérer de concevoir le déroulement de leurs cours suivant le même type de schématisation, et pour le même

\footnotetext{
17 Novice au sens où il n'était pour l'enseignant du cours de 2012-13 (un des co-signataires de l'article) qu'une première tentative imitée de l'année précédente.

18 Cette réforme est celle qui a été initiée conjointement par les ministères de l'éducation nationale et de l'enseignement supérieure et entérinée par deux lois, l'une sur la « refondation de l'école» (publiée au JO en juillet 2013), l'autre sur l'enseignement supérieur et la recherche (loi ESR, parue au JO le 23.7.13). Elle cherchait à pallier les défauts d'une précédente réforme initiée à partir de 2008 et mis en œuvre à partir de 2009-10 sous le précédent gouvernement.

${ }^{19}$ En fait la chose serait possible en s'intéressant à un nouveau cours du même enseignant, dont le contenu n'était pas directement historique mais touchait à la connaissance du contexte institutionnel de l'enseignement secondaire. Un schéma semblable permettrait de repérer facilement la parenté d'inspiration avec les deux cours en question.

${ }^{20}$ Le principe de cette formation à l'enseignement pour doctorants contractuels est décrit plus en détail dans le billet 510 du carnet STI.
} 
usage réflexif. Néanmoins, on peut raisonnablement penser que ce type de travail est susceptible de leur donner des pistes pour l'élaboration de conception globale ${ }^{21}$.

Arrêtons nous maintenant sur un autre critère intéressant pour sa fécondité, à savoir celui que nous avons primitivement intitulé « dossier documentaire ».

\subsection{La réflexion évolutive autour du dossier documentaire proposé aux étudiants de ce type de cours}

D'après la définition qui en a été proposée formellement après la 1ère séance de séminaire, il s'agit d'une «Archive documentaire mise à disposition des étudiants, pour baser leur travail réflexif, en indexant cette archive par les différentes informations qui permettent d'en situer les usages pédagogiques, qu'il soient intentionnels ou effectifs. »Ce qu'on essaie de formaliser ici est une pratique courante qui se retrouvait dans les trois cours comparés, et en général dans de très nombreux cours du même type puisqu'ils ont l'ambition de conduire à une lecture autonome de textes historiques. Puisqu'une place est généralement laissée à la préparation d'un dossier d'étude par l'étudiant et choisi par ce dernier, qu'il soit présenté pendant le cours, à la fin de ce dernier ou le plus souvent à la fois pendant et à la fin (si des travaux intermédiaires sont demandés), le problème se pose de définir le point de départ de cette enquête pour les étudiants ${ }^{22}$. Ce point de départ peut-être fourni de multiples manières plus ou moins formalisées.

A titre d'exemple, on trouvera en annexe $5[\underline{x}]$ la copie d'écran du dossier documentaire mis à disposition pour l'année 2011-12 des étudiants du master MFTIGS. Il s'agit d'un dossier constitué d'une arborescence très simple et accessible via la plateforme de cours en ligne accessible aux étudiants et collaborateurs du cours ${ }^{23}$. Chaque dossier donne accès à un ou plusieurs documents de natures diverses, depuis une copie d'écran d'un site existant, à une synthèse rédigée par un enseignant, ou bien une collection d'articles puisés à différentes sources et rendant compte d'un débat sur un sujet donné. La discussion en séminaire sur ces dossiers et sur le suivi des différents travaux d'étudiants encore lisibles, a fait apparaître d'emblée un problème important, à savoir qu'il était difficile de concilier le choix autonome d'un sujet d'exposé ou de travail par l'étudiant, l'idée de guider leurs lectures de départ, et enfin la constitution collaborative de ces dossiers ${ }^{24}$.

Il était en effet dans l'intention des concepteurs de ce cours, de permettre aux étudiants de choisir un sujet qui soit le plus près possible de leur intérêt et donc de les laisser libre du thème à traiter dans leur dossier. Mais en première année de master, il semblait difficile de les laisser totalement autonome sur la recherche des ressources au vu de la durée du module, d'où la préconception d'un certain nombre de dossiers documentaires proposant des thèmes assez divers pour permettre un choix. Ces dossiers très proches de leurs compétences disciplinaires touchaient donc des thématiques très variées. Ils ont par conséquent nécessité un travail de tentatives de collaboration sur plusieurs mois avec les enseignants des disciplines scientifiques et techniques du master. Ces collaborations n'ont pas été toujours été faciles mais ont au moins permis de montrer aux enseignants en question en quoi consistaient ce module et son intérêt didactique et professionnel pour ces futurs enseignants.

Par la suite, et largement en raison des évolutions du master en question, il n'y a malheureusement pas eu d'effet "boule de neige" qui aurait pu pousser l'équipe enseignante à renforcer cette coopération

\footnotetext{
21 Cette remarque a d'autant plus d'importance que bien des doctorants, dans le cas favorable où ils sont recrutés rapidement, sont mis en demeure de concevoir des cours entiers, sans recevoir pour autant de formation ou même de simples conseils sur la manière d'organiser de manière cohérente leurs cours.

${ }^{22}$ Ce repère heuristique est discuté en détail dans le billet de synthèse $n^{\circ} 294$ du carnet STI, sous la rubrique «le problème des documents mis à disposition des étudiants ».

23 Il s'agit d'une plateforme développée à partir de la plateforme Claroline depuis 2001 par des collègues de l'Université catholique de Louvain (UCL) dans le cadre d'un consortium du même nom.

24 Voir les observations plus développées proposées dans l'annonce de la dite séance, billet 125 du carnet STI.
} 
naissante et à proposer par exemple de nouveaux dossiers, ou à modifier les anciens. L'effet positif a finalement surtout été obtenu auprès des étudiants, qui ont pu mieux identifier les connexions entre cet enseignement, et leurs enseignements disciplinaires. Par contre, l'explicitation de cette réflexion a vite conduit à une modification des pratiques dans d'autres cours. A titre d'exemple, le cours d'histoire des mathématiques dont il a été question plus haut (\$2.2) comportait, lui aussi, un dossier documentaire puisque sa structure imposait une démarche par projets impliquant un important travail de préparation en bibliothèque et la soutenance d'exposés avec remise d'un travail écrit en fin de module. Le premier dossier documentaire (année 2011-12, schéma en annexe 2) consistait en une suite de textes proposés en format pdf formant volontairement une mosaïque disparate: il était laissé à la charge des étudiants d'enrichir le commentaire par des recherches documentaires complémentaires. Dans la version suivante, l'arborescence a été perfectionnée pour inclure différents niveaux de lecture possible du dossier, suivant le degré d'autonomie que l'étudiant était prêt à prendre avec le choix de son sujet. Chaque texte ou lien était ainsi codé suivant la nomenclature suivante:

- EXTR = extrait d'un texte, suivant le même format que les textes donnés en TD.

- PRIM COMP : source primaire complète, généralement donnée par un lien vers un site fiable de ressources numérisées (comme Gallica, ou Bibnum).

- SEC COMP : source secondaire complète mise en ligne là encore sur des sites fiables, comme revues.org.

- SITE : un site sur lequel on trouve toutes sortes de textes primaires ou secondaires, comme par exemple CultureMATH (site expert de l'ENS).

Par ailleurs l'arborescence des dossiers permettait aux étudiants de repérer de grands domaines d'intérêt liés à des catégories disciplinaires classiques en mathématiques (voir annexe 6 [ㅈ]).

Le point important est de constater l'évolution des outils en fonction de la réflexion commune: une fois reconnu explicitement le problème posé par la façon de négocier l'équilibre entre accompagnement et autonomie des étudiants, il devenait possible en retour d'améliorer les dossiers existants pour tenir compte de cette reconnaissance.

Au total, l'analyse détaillée présentée ici, permet de se représenter l'esprit et d'afficher la dynamique à l'œuvre lors des réunions de ce groupe d'enseignants. Les deux dernières parties (1.2 et 1.3) ont illustré notamment l'évolution et la stabilisation relative des pratiques, grâce à leur explicitation au sein du groupe. Pour permettre une lecture plus approfondie et détaillée de la dynamique en question et ce qui l'a rendue possible, nous proposons maintenant une première théorisation de cette expérience en rappelant les constituants fondamentaux des communautés de pratique, ainsi que la raison d'être de ces dernières.

\section{Un cadre d'analyse possible pour ce type de coopération : les communautés de pratique centrées sur l'innovation pédagogique en milieu universitaire}

\subsection{Les communautés de pratique : rappel du cadre général}

Dans toute situation où un groupe de praticiens est engagé dans une activité bien identifiée pour ses membres, que cette activité soit de caractère professionnel ou autre, mais sans que cette collectivité née d'une pratique émergente n'ait de reconnaissance institutionnelle directe, la notion de communauté de pratique s'avère pertinente. En effet, comme le rappelle à juste titre Amaury Daele dans une synthèse sur cette question (Daele 2009) le caractère «émergent » et naturaliste de la notion a été construit pour penser des situations où des groupes se structuraient autour de pratiques partagées, qui avaient pour les membres une valeur forte de « socialisation ». Il nous semble ainsi que les groupes d'universitaires partageant une pratique d'enseignements en sciences humaines, quand du moins celle-ci est en situation 
de relative marginalité par rapport aux cursus de sciences et techniques qui les accueillent, peuvent bénéficier de la théorie des communautés de pratique notamment dans la déclinaison particulière qu'elle a prise récemment pour décrire le développement de communautés d'enseignants universitaires autour de pratiques pédagogiques innovantes ${ }^{25}$ (Daele 2009 §1.2).

Si l'on s'en rapporte à l'exposé classique de ce qui caractérise et permet de favoriser le développement de communautés de pratique (Wenger and Snyder 2002), les communautés de pratique sont définies comme «des groupes de personnes qui partagent un intérêt commun, un ensemble de problèmes, ou encore une passion sur un sujet donné, et qui approfondissent leur connaissance et leur expertise dans ce domaine en interagissant entre eux d'une manière prolongée » (ibid. p.4). Le premier point fondamental à retenir de cette définition est celui de la préexistence de la « raison d'être ensemble » de ces groupes ; une communauté de pratique n'est pas créée ex nihilo, elle s'appuie toujours sur un partage d'intérêts et de pratiques existantes. Le second est celui du développement naturel. Une communauté de pratique est comparable à une structure vivante qui possède son propre principe de croissance et de développement : sa pérennité n'est ainsi jamais garantie et son développement dépend des circonstances qui lui sont favorables ou non. Le principe d'interaction avec un milieu est donc essentiel à la notion. Enfin il faut retenir la centralité de l'apprentissage et de l'innovation (learning) dans la raison d'être d'une communauté de pratique: elle vise au développement d'outils ou de connaissances nouvelles.

Du point de vue analytique déjà proposé par Wenger dans sa première théorisation de la notion, les trois constituants fondamentaux d'une communauté de pratique sont l'existence d'un domaine d'intérêt commun, d'une communauté effective et enfin d'une pratique partagée. En conformité avec le caractère fondamentalement vivant intrinsèque à la définition des communautés de pratique, aucun de ces éléments ne devrait être considéré comme figé ou défini de manière univoque. Il s'agit plutôt à chaque fois, d'un aspect fondamental mais qui reste ouvert à renégociation et à une évolution constante au cours de l'existence de la communauté concernée. Ainsi le domaine d'intérêt est-il susceptible d'évoluer; les modalités de fonctionnement collectif également; de même que la nature des pratiques partagées $^{26}$.

Revenons alors sur l'exemple proposé plus haut de notre groupe d'enseignants en regardant à nouveau son évolution, et l'élaboration de pratiques et d'objets nouveaux, à l'aune de ces points de repères théoriques. Nous nous proposons ainsi de relire l'évolution du groupe et de ses pratiques, en nous focalisant sur l'évolution du domaine, des pratiques et de la communauté en question.

\subsection{Les conditions du maintien et de l'évolution d'une communauté}

Qu'une communauté telle que celle que nous avons plus haut décrite, ait pu se constituer et se maintenir dans le temps, ne relève pas de l'évidence. En France, en effet, elle est généralement sujette au problème endémique de toute communauté de réflexion sur les pratiques pédagogiques et leurs enjeux. Comme les pratiques collectives visant à améliorer la meilleure professionnalisation des enseignants ne font jusqu'ici que marginalement partie des statuts et de la formation des enseignants universitaires, ce type de communauté reste précaire et difficile à fédérer. Dans le cas particulier des enseignements de sciences humaines et sociales dans et pour la formation des étudiants en sciences et techniques, il existe des sociétés savantes particulières qui soutiennent des échanges ponctuels dans le cadre de leurs colloques, mais pas encore de structure plus globale semblable aux communautés actives et publiantes réunies

\footnotetext{
25 D'abord à l'étranger puis plus récemment en France.

26 Cette mobilité essentiel de tous les aspects d'une communauté de pratique n'est facteur de destruction, relèvent Wenger, Snyder et McDermott, que lorsque les trois sont en renégociation: mais dans un développement fécond et pérenne, la possibilité de renégociation d'un aspect (par exemple, un questionnement sur le domaine de référence) repose sur la stabilité d'un autre (par exemple, des pratiques communes ou des règles de fonctionnement collectif stabilisées) (p. 47).
} 
sous la bannière du « scholarship of Teaching and Learning » (SoTL) qui, dans les pays anglo-saxons par exemple, ont permis l'émergence de rencontres régulières et de lieux de publications académiques officialisant et soutenant la réflexion pédagogique de niveau universitaire (Colet et al. 2011).

Paradoxalement, concernant les enseignements de sciences humaines dans les cursus scientifiques et techniques, le fait même qu'ils soient souvent considérés comme plus ou moins marginaux par rapport au cœur de la formation, invite à davantage de collaboration. Ils sont en effet construits et maintenus par des enseignants qui sont et/ou se sentent isolés en tant qu'enseignants et chercheurs en sciences humaines et qui sont le plus souvent contraints d'innover pour adapter leur enseignement au contexte particulier d'une formation spécialisée ${ }^{27}$.

Quoiqu'il en soit, la première condition d'existence d'une telle communauté réside donc dans la possibilité concrète de maintenir un espace et un temps dédié à des rencontres sur ce type de sujet. Dans le cas qui nous intéresse, si on récapitule les observations ci-dessus $(\S 1.1)$, les séminaires du groupe « interférence » ont permis cette structuration temporelle du groupe et jouent un rôle crucial dans le maintien de la communauté comme dans son développement par son ouverture à des participants potentiellement concernés. Corrélativement, le carnet de recherche associé est devenu un élément très important de formalisation, de mémoire et de communication des réflexions de cette communauté. Tout ceci reposait encore sur l'existence, classique là encore dans le maintien de communautés de pratique, de coordinateurs, de collègues pouvant jouer le rôle de passeurs ou de catalyseurs pour l'élaboration de collaborations concrètes. On a vu enfin que la mise en place du projet de recherche associé tenait à des raisons institutionnelles fortes qui lui permettaient d'exister.

Il faut néanmoins remarquer que si tout ceci représente un ensemble de conditions nécessaires au maintien de la communauté, il ne suffit pas à en caractériser la clé de voûte, à savoir le projet de recherche lui-même. La question déterminante, concrètement posée à titre de critère de sélection des thématiques des séances de séminaires, était l'intérêt que tous les membres pouvaient y porter en fonction d'un projet commun et fédérateur. Les réunions qui ont visé à consolider la première année de séminaire, en 2012, ont conclu à la nécessité de ne pas « spécialiser » les séances sur des thématiques particulières, mais de faire un choix qui permette à chaque fois une nouvelle discussion de l'argumentaire du projet dans son ensemble. Autrement dit, de créer un lien intrinsèque entre l'évolution du domaine d'intérêt commun, et celui de la communauté elle-même.

\subsection{La définition évolutive d'un domaine d'intérêt commun}

On vient de voir que ce qui tenait et tient cette petite communauté est le partage d'une problématique fédératrice. Plus précisément, le fait de comparer de manière dynamique des enseignements de sciences humaines dans différentes formations pour enseignants de sciences et techniques, était une façon d'explorer une des questions cardinales de ce projet: comment s'hybrident des compétences ou des champs de recherche divers, qui participent à la circulation, la production et la transmission de savoirs scientifiques et techniques? La définition précise du projet, notamment de son second axe consacré au départ aux enseignements universitaires de sciences humaines, évolue : alors qu'elle ne s'attachait tout d'abord qu'à la comparabilité d'enseignements, c'est aussi à la question de la constitution collaborative de ressources et de références partagées que le groupe s'intéresse désormais, comme faisant un tout. La réflexion qu'on a résumée plus haut, sur les dossiers documentaires, est précisément ce point de passage qui montre qu'il est difficile de séparer la conception d'un cours conçu à plusieurs, avec l'élaboration d'une ressource documentaire partagée et donc construite elle aussi de manière collaborative. On a

\footnotetext{
${ }^{27}$ Les deux phénomènes sont liés, puisque les collègues en question doivent consacrer une partie conséquente de leur temps à mettre en place et défendre les enseignements en question. Voir sur ce point l'intervention de Michel Cottes, dans ce volume «L'organisation des S.H.S. en écoles d'ingénieurs, deux exemples opposés » ainsi que la contribution de Hourcade « Entre culture générale et enjeux des métiers d'ingénieur : la diversité des enseignements en sciences humaines et sociales à l'école centrale de Lyon » dans ce volume. C'est autant de temps pris sur celui qu'ils pourraient investir sur des programmes de recherche ambitieux.
} 
donc bien affaire ici à une communauté d'intérêt, intrinsèquement attachée à la définition d'un projet de recherche, et au fait que cette dernière est évolutive.

Mais si l'on reste à la comparaison des enseignements en question, d'autres facteurs originaires ont également joué. La comparabilité possible de ces enseignements tenait en effet, en premier lieu, à ce qu'ils avaient profité initialement d'un espace de concertation qui avaient poussé trois d'entre nous à définir en 2008 la raison d'être de tels enseignements dans les nouveaux masters qui se mettaient alors en place. L'annexe $7[\underline{\mathrm{x}}]$ montre le document qui avait été réalisé, et qui se positionnait résolument suivant une sorte d'approche programme, déclinant les objectifs de ces formations, en fonction des compétences professionnelles à développer chez les enseignants. De ce point de vue, l'une des conditions de possibilité du domaine d'intérêt commun était l'existence désormais inscrite dans la durée, de la politique universitaire d'inspiration européenne qui, avec la structuration en licence-masterdoctorat (LMD), invite à développer toujours plus de lisibilité des formations pour les étudiants comme pour les professionnels ou les enseignants eux-mêmes. C'est notamment cette tendance lourde qui invite à privilégier des formes d'interdisciplinarité visant à développer des compétences transversales, que ce soit en licence (pour privilégier leur caractère généraliste) ${ }^{28}$ ou dans les masters vers l'enseignement. Il s'agit dans cette optique de promouvoir une forme large de culture scientifique, mais aussi des compétences professionnelles particulières comme la recherche de ressources et leur validation, le positionnement épistémologique d'une science envers les autres sciences et ce qui en découle en terme d'enseignement, l'aptitude à un regard critique sur le développement scientifique en développant une aptitude à le repositionner vis à vis de la politique, de l'économie, de l'histoire et des débats de sociétés ${ }^{29}$. A cet égard, donc, la définition d'un domaine d'intérêt commun tient en partie à la possibilité de faire référence à ce type de référentiels essentiellement tributaires de la politique universitaire et de ses évolutions récentes.

Enfin on peut discerner dans le cas d'étude présenté plus haut, une raison plus subtile pour non seulement maintenir mais (surtout) faire évoluer concrètement le domaine d'intérêt commun. Nous faisons allusion aux étapes de constitution et d'évolution des dossiers documentaires, qui engagent profondément, à elles seules, les questions de négociation du domaine. A partir du moment en effet où l'on constitue ce type de dossier, « on » signifiant ici généralement une équipe d'enseignants au sein de laquelle chacun contribue ${ }^{30}$, on mutualise implicitement une connaissance du champ au travers des documents choisis, de la manière de les présenter ou de les organiser. On peut donc s'attendre à ce qu'il y ait un effet rétroactif sur la définition du champ concerné et sur ses marges. Cette problématique, qui ne concerne pas que les cours de master, a débouché, dans le cas de notre communauté, sur une première volonté de mutualiser des références bibliographiques par des outils de partage appropriés (du type de Zotero). Si l'expérience n'est pas encore assez avancée pour autoriser des conclusions détaillées, elle permet néanmoins d'entrevoir le type de pratiques partagées que l'existence de la communauté en question a permis ou permet d'envisager.

\subsection{L'élaboration de pratiques partagées}

L'élaboration de démarches intellectuelles et pédagogiques partagées dans le cadre qui nous intéresse, oblige tout d'abord à une remarque préalable sur ce qui motive ce travail commun. On a vu en effet plus haut que les pratiques d'enseignement en sciences humaines, à partir du moment où il faut justifier leur raison d'être dans le cadre de référentiels proposés pour identifier les formations universitaires,

\footnotetext{
28 Voir le référentiel des compétences en licence (2012) et la loi ESR de 2013.

29 Exemple : BO de 2011 sur la promotion de la culture scientifique.

30 Que cet effort soit autant que possible collectif présente des avantages évidents dans le cadre du renforcement de la cohérence d'une formation: plus les étudiants travaillent plus ou moins sur les mêmes documents sous différents points de vue, plus on peut s'attendre à un gain en cohérence.
} 
conduisent assez souvent à privilégier les compétences transversales dans les référentiels en question, comme par exemple l'acquisition d'une culture générale dans le champ scientifique, la compréhension des enjeux associés, l'autonomie dans le travail, la capacité de recherches d'informations, d'analyse et de synthèse, la maitrise de l'expression écrite et orale. Ces objectifs, d'un côté, tendent à guider les pratiques vers des dénominateurs communs et de l'autre obligent aux confrontations. En effet le caractère souvent transversal des objectifs et compétences visées se conjugue à l'absence relative d'un corpus de connaissances, de documents ou de dispositifs qui seraient privilégiés pour les développer. Au contraire, l'extrême variabilité de ces références et des pratiques possibles pour atteindre les objectifs explique suffisamment que de larges espaces de renégociation et de mutualisation soient ici ouverts.

Dans l'expérience concrète de collaboration autour de la conception de plusieurs cours qu'on a rapportée plus haut, les pratiques innovantes qui autorisaient cette comparaison et qui ont retenu l'attention du groupe étaient, comme on l'a vu, des manières d'élaborer des formes de visualisation efficaces, qu'il s'agisse de schématisations globales du scénario d'un cours et du travail des étudiants associé, ou des arborescences caractérisant le dossier documentaire mis à leur disposition. L'enjeu principal était de concevoir les moyens d'entretenir une communication à la fois rapide mais efficace et profonde, permettant non seulement de comparer des conceptions mais encore d'en construire de nouvelles; à ce titre, elle a conduit comme on l'a vu à des innovations pédagogiques au niveau de la formation des doctorants.

Au niveau de nos pratiques pédagogiques elles-mêmes, on peut relire certaines des remarques faites plus haut sur les « repères heuristiques » comme autant d'indicateurs d'évolution partagée de nos pratiques, à commencer par le questionnement qui motivait les changements souhaitables. A titre de premier exemple, on retrouve la problématique centrale qui nous conduisait à visualiser le travail des étudiants, ainsi que le dossier documentaire associé : il s'agissait bien, au fond, de prendre acte de la manière d'accompagner et de guider un travail tout en invitant l'étudiant à devenir autonome, pour reposer le problème délicat du choix du sujet et de son rapport à la base documentaire proposée. Dans le fond, c'est le partage de cette question qui a conduit à faire porter une partie de la réflexion sur la lisibilité de l'arborescence proposée aux étudiants.

L'autre exemple a été rapporté plus haut : la réflexion sur la construction d'une documentation pour les étudiants, nous a conduits à la question plus large de la constitution d'une référence commune, sous forme par exemple d'une archive ou bien d'une bibliothèque élaborée collaborativement. On glisse ici, à nouveau et sans solution de continuité, depuis la comparaison des méthodes pédagogiques vers des mutualisations de pratiques.

\section{Les conclusions à tirer de cette modélisation d'un développement collaboratif d'enseignements universitaires}

L'analyse qui précède montre qu'une relecture du développement d'une communauté d'enseignants localisée et de ses pratiques à travers le prisme de la théorie des communautés de pratique est possible. On peut non seulement reconnaître dans cette expérience les trois fondamentaux d'une communauté de pratique, un domaine d'intérêt commun, des pratiques partagées et un fonctionnement collectif effectif, on a vu aussi que chacun de ces pôles avait une dynamique propre et «bougeait ». La question qui doit alors nous intéresser logiquement est celle de l'intérêt prospectif de cette analyse fondée sur le choix d'un modèle, pour la réflexion sur le devenir de ce groupe et de son travail commun. L'un des intérêts de l'utilisation de ce cadre des communautés de pratique est certainement de prendre conscience des aspects structurants mais aussi de la fragilité des communautés locales d'enseignants telle que celle décrite ici.

Il faut rappeler que le concept de communauté de pratique est depuis sa création le porteur d'une ambigüité fondamentale. D'un côté, il est conçu pour décrire et identifier un mode d'apprentissage collectif émergent et présuppose toujours que les communautés visées existent naturellement sous 
des formes très variées mais néanmoins identifiables. Pour l'essentiel, c'est à cette description que nous nous sommes livrés dans la première partie, et dans une certaine mesure dans la seconde. D'un autre côté, ces communautés peuvent être envisagées sous des aspects prospectifs, le problème fondamental étant d'étudier les conditions institutionnelles pour que de telles communautés puissent se développer et perdurer dans des conditions favorables, ou si au contraire il est temps pour elles de mourir et/ou d'essaimer. Les métaphores biologiques sont souvent privilégiées pour décrire la nature et le développement des communautés de pratique: si ces dernières sont des arbres ayant leur vie propre, le problème est d'étudier les conditions pour que cet arbre trouve un milieu et des nutriments favorables à sa croissance ou à sa dissémination pour essaimer dans de nouvelles communautés.

$\mathrm{Si}$ on revient alors aux questions de milieu, la première grande conclusion à tirer de l'analyse précédente (partie 2) est qu'on peut identifier les conditions institutionnelles qui ont permis ce développement, sans que ces communautés aient une existence reconnue institutionnellement. Ces conditions, en résumé, sont globales et renvoient à la continuation sur le long terme de la politique de mise en conformité des cursus universitaires de niveau licence, master ou doctorat, avec un modèle européen où l'accent est mis sur la lisibilité des formations, et l'identification explicite de leurs objectifs. Il en résulte une incitation générale au développement des enseignements permettant de cultiver chez les étudiants un ensemble riche de compétences. Mais elles sont aussi locales puisque le travail présenté est fondé essentiellement sur la mise en place de nouvelles institutions, comme les ESPE, ou de nouvelles formations, comme celles de niveau master pour la formation des futurs enseignants, dont certains aspects nous ont servi ici de «terrain d'étude ». Ce contexte explique alors une observation fondamentale sur les évolutions en cours, à savoir que la demande de transversalité et de cours " généralistes », à laquelle répondent le type de cours décrit ici, ne fait que s'accentuer et tout particulièrement au niveau des premières années de formation supérieure, au niveau licence (1ère année de licence, 1ère année en école d'ingénieur ou en médecine).

Cette pression globale pose immédiatement la question des forces de formation disponibles ${ }^{31}$. Les observations faites plus haut sur le « glissement naturel » d'une pratique de mutualisation de pratiques pédagogiques entre pairs, vers des opérations de formation de nouveaux venus, prend alors un sens nouveau: ce type de formation pourrait devenir la condition d'un recrutement raisonné de vacataires puis de collègues pour non seulement répondre à une certaine demande, mais le faire de manière concertée, au sein d'une équipe partageant des pratiques et ressources communes. Il renforce de même le bien fondé du projet de construire des moyens efficaces pour comparer et surtout élaborer de nouveaux enseignements. L'expérience décrite ici ne serait alors qu'une préfiguration de ce que nous aurions besoin de mettre en place pour structurer une offre pédagogique cohérente, fondée sur une réflexion amont.

La seconde conclusion de notre analyse pointe vers l'importance de pouvoir fonder le travail non seulement sur des rencontres, mais encore sur un projet de recherche évolutif, souple. On a vu en effet que le maintien de la communauté qui a produit le travail dont on a résumé ici certains aspects, n'est pas dissociable de la définition et de l'évolution de son domaine d'intérêt. On peut se demander si l'existence d'un tel projet fédérateur, qui n'entre dans aucun champ de recherche en particulier mais auquel plusieurs contribuent, n'est pas une condition pour que se développe ce type de problématique. Son caractère évolutif joue ici un rôle, puisqu'il justifie en profondeur la tenue d'un séminaire où puisse, entre autres choses, être débattues ce type de questions et l'évolution de la problématique visée. On retrouve ici un problème plus généralement abordé, outre-Atlantique, au sujet du SoTL (Scholarship of Teaching and Learning) : comment combiner l'activité de recherche et d'enseignement des collègues impliqués ?

\footnotetext{
31 A titre d'exemple, certains membres de notre groupe ont élaboré une proposition de cours transversaux d'histoire des sciences et des techniques pour tous les cursus de sciences et technologies de l'université. Le point intéressant est que si cette proposition était acceptée, nous serions immédiatement en sous-effectifs pour en assurer la mise en œuvre.
} 
Ce problème est d'autant plus crucial qu'il doit désormais tenir compte d'une nouvelle observation, à savoir que l'offre de formation portant sur la pédagogie universitaire se développe à grande vitesse au niveau de l'université comme du PRES. A titre d'exemple, une initiative s'est développée récemment pour promouvoir au niveau de l'UPEC la mutualisation de la réflexion pédagogique. Fondé explicitement sur le concept de communauté de pratique, le groupe d'enseignants qui a pris en charge l'animation de ces ateliers est déjà fort de plus d'une vingtaine de membres, dont plusieurs membres du groupe dont il a été question ici, et deux des cosignataires de cet article.

Une différence essentielle subsiste cependant entre cette nouvelle communauté de pratique uniquement centrée sur des questions de pédagogie universitaire, sans lien fort (pour l'instant) à des projets de recherche, à la réflexion ici présentée dont le lien à une problématique de recherche est essentiel. Il faut ici prendre en compte une des conclusions de l'analyse précédente sur l'évolution de nos pratiques partagées. Nous nous sommes en effet aperçus qu'il y avait toutes les raisons de construire un lien fort entre mutualisation voire création de ressources en sciences humaines, et ingénierie pédagogique. C'est le problème profond auquel renvoie le critère du « dossier documentaire »: en partant d'une « simple » réflexion pédagogique, on débouche sur une redéfinition plus globale du champ, non plus seulement au travers de la définition d'une problématique commune, mais au travers de la digestion collective de références communes et mutualisées activement. Ce saut, d'un geste pédagogique vers une problématique de recherche, ressemble à ce que décrivent $\mathrm{C}$. Bonah et ses collaborateurs, dans ce même volume.

Enfin et plus généralement, revenons au problème formulé en introduction, concernant l'existence de communautés locales et relativement isolées d'enseignants en charge du type d'enseignement en sciences humaines et sociales dont il a été question ici. Peut-on tirer des conclusions générales des réflexions précédentes ? D'un côté, il est évidemment difficile d'entrer dans de telles généralités, qui rejoignent à certains égards la problématique du colloque dans son entier. De ce point de vue, la démarche la plus pertinente est d'accumuler les études de cas pour faire des comparaisons entre des contextes institutionnels et des domaines de formation différents. D'un autre côté, on peut se demander ce que sont les ressources et appuis à privilégier pour développer ce type de collectif. Si cet appui doit passer par un effort de recherche, comme cela a été le cas pour nous, alors la question est peut-être de savoir comment cette recherche intègre les questions pédagogiques dans un ensemble problématique plus vaste, et pourquoi. Le modèle des communautés de pratique a paru ici intéressant pour faire le pont entre des problématiques d'ordre pédagogique et d'autres questions plus vastes, est-ce pourtant le seul à pouvoir le faire ? Cette question reste ouverte.

\section{Références}

Bonah, Christian. 2006. «L'enseignement Des Sciences Humaines et Sociales En Médecine ? : Éléments Pour Un Panorama de La Situation En France. »In Médecine et Sciences Humaines. Sciences Humaines En Médecine? : Formation et Collaboration, 9-18. Berne : Académie Suisse des sciences humaines et sociales.

Colet, Nicole Rege, Lynn McAlpine, Joëlle Fanghanel, and Cynthia Weston. 2011. " Le concept de Scholarship of Teaching and Learning. » Recherche et formation, no. 67 (May): 91-104. doi:10.4000/rechercheformation.1412.

Collège des enseignants de SHS en médecine et santé. 2011. Médecine, santé et sciences humaines: manuel. Médecine \& sciences humaines 17. Paris : les Belles lettres.

Daele, Amaury. 2009. «Les communautés de pratique. » In Encyclopé die de la Formation, edited by Jean-Marie Barbier, Étienne Bourgeois, Gaëtane Chapelle, and Gilles Marchand. Paris, France : Presses universitaires de France, DL 2009.

Guedj, Muriel. 2006. « Avant-propos. » Tréma, no. 26 (October) : 1-3. 


\section{SHS Web of Conferences}

\section{Annexes}

Voici la liste des sept annexes de l'article, le lien correspondant permettant à chaque fois d'accéder au document. Les annexes sont également récapitulées dans le billet 670 du carnet STI [ $\underline{x}]$.

1. Organisation synoptique de l'UE d'histoire et d'épistémologie des sciences du master MFTIGS, 2011-12. [ㅈ]

2. Organisation synoptique d'un cours d'histoire des mathématiques en master, 2011-12 (document élaboré en avril 2013). [X]

3. Organisation synoptique du même cours d'histoire des mathématiques, 2012-13 (élaboré en Jan. 2013). [] $]$

4. Synopsis du cours du 2011-12, comportant une indication sur les compétences travaillées. [X]

5. Dossier documentaire du cours EHST du master MFTIGS, 2011-12 [ㅈ p.1]

6. Dossier documentaire du cours d'histoire des mathématiques dont l'organisation est décrite en annexe 3 (2012-13) [ㅈ p.2]

7. Texte de proposition pour des formations en épistémologie et histoire des disciplines d'enseignement scientifiques et technologiques, dans le cadre des masters et parcours de masters « enseignement » (2008, A. Mayrargue, A.S. Génin, A. Bernard) [X] 